\title{
Use of complex visual stimuli allows controlled recruitment of cortical networks in infants
}

\section{Ahtola, Eero}

2020-04-23

Ahtola , E , Stjerna , S , Tokariev , A \& Vanhatalo , S 2020 , ' Use of complex visual stimuli allows controlled recruitment of cortical networks in infants ', Clinical Neurophysiology , vol. 131 , no. 8 , pp. 2032-2040 . https://doi.org/10.1016/j.clinph.2020.03.034

http://hdl.handle.net/10138/329313

https://doi.org/10.1016/j.clinph.2020.03.034

cc_by_nc_nd

acceptedVersion

Downloaded from Helda, University of Helsinki institutional repository.

This is an electronic reprint of the original article.

This reprint may differ from the original in pagination and typographic detail.

Please cite the original version. 


\section{Journal Pre-proofs}

Use of complex visual stimuli allows controlled recruitment of cortical networks in infants

Eero Ahtola, Susanna Stjerna, Anton Tokariev, Sampsa Vanhatalo

PII:

S1388-2457(20)30141-3

DOI:

https://doi.org/10.1016/j.clinph.2020.03.034

Reference:

CLINPH 2009200

To appear in: $\quad$ Clinical Neurophysiology

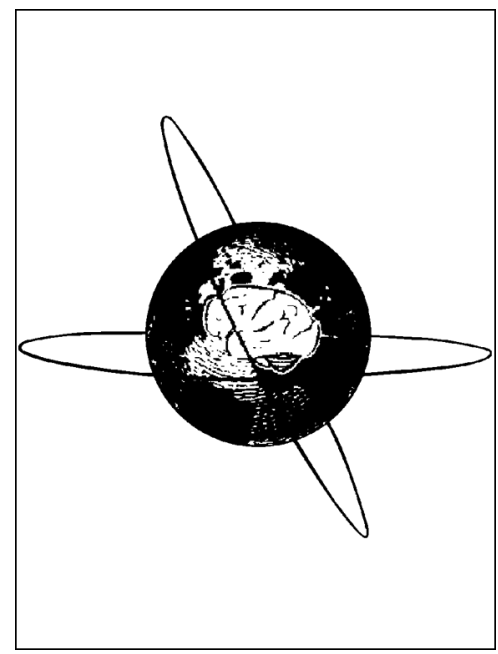

Received Date: $\quad 10$ September 2019

Revised Date: $\quad 25$ February 2020

Accepted Date: $\quad 16$ March 2020

Please cite this article as: Ahtola, E., Stjerna, S., Tokariev, A., Vanhatalo, S., Use of complex visual stimuli allows controlled recruitment of cortical networks in infants, Clinical Neurophysiology (2020), doi: https:// doi.org/10.1016/j.clinph.2020.03.034

This is a PDF file of an article that has undergone enhancements after acceptance, such as the addition of a cover page and metadata, and formatting for readability, but it is not yet the definitive version of record. This version will undergo additional copyediting, typesetting and review before it is published in its final form, but we are providing this version to give early visibility of the article. Please note that, during the production process, errors may be discovered which could affect the content, and all legal disclaimers that apply to the journal pertain.

(C) 2020 International Federation of Clinical Neurophysiology. Published by Elsevier B.V. All rights reserved. 


\section{Use of complex visual stimuli allows controlled recruitment of cortical networks in infants}

Authors: Eero Ahtola ${ }^{1,2}$, Susanna Stjerna ${ }^{1}$, Anton Tokariev ${ }^{1,3}$, Sampsa Vanhatalo ${ }^{1,3}$

Affiliations:

1. BABA Center and Department of Clinical Neurophysiology, Children's Hospital, Helsinki University Hospital and University of Helsinki, Helsinki, Finland

2. Department of Neuroscience and Biomedical Engineering, Aalto University School of Science, Espoo, Finland

3. Neuroscience Center, Helsinki Institute of Life Science, University of Helsinki, Helsinki, Finland

\section{Corresponding Author:}

Eero Ahtola

Department of Clinical Neurophysiology, Children's Hospital, Helsinki University Hospital

Stenbäckinkatu 9, 00029 HUS, Helsinki, Finland.

Email: eero.ahtola@hus.fi, tel. +358503630810.

\section{Abstract}

Objective: To characterize cortical networks activated by patterned visual stimuli in infants, and to evaluate their potential for assessment of visual processing and their associations with neurocognitive development.

Methods: Three visual stimuli, orientation reversal (OR), global form (GF), and global motion (GM), were presented to cohort of five-month-old infants $(\mathrm{N}=26)$. Eye tracker was used to guide the stimulation and to choose epochs for analysis. Visual responses were recorded with electroencephalography and analysed in source space using weighted phase lag index as the connectivity measure. The networks were quantified using several metrics that were compared between stimuli and correlated to cognitive outcomes.

Results: Responses to OR/GF/GM stimuli were observed in nearly all (96/100/100\%) recordings. All stimuli recruited cortical networks that were significantly different in their properties. The more complex GF and GM conditions recruited wider global networks than OR. Additionally, strength of the GF network showed positive association with later cognitive performance.

Conclusions: Network analysis suggests that visual stimulation recruits vast cortical networks extending far beyond the conventional visual streams and differ between stimulation conditions.

Significance: The method allows controlled recruitment of wide cortical networks, which holds promise for the early assessment of visual processing and its related higher-order cognitive processes.

\section{Keywords}

Visual Evoked Response, Visual System, Infant, Brain Connectivity, Functional networks, Neurodevelopment

\section{Highlights}

- Visual stimulation recruits wide cortical networks in infants.

- Network organization varies with the type of visual stimulus.

- Quantitative descriptors of these networks correlate with later cognitive performance. 


\section{Abbreviations}

BSID-III, Bayley Scales III; CC, clustering coefficient; CN, consistent network; CN wPLI, average connectivity within a consistent network; $C P L$, characteristic path length; $E E G$, electroencephalography; $f$, stimulation frequency; FDR, false discovery rate; GF, global form; GM, global motion; MRI, magnetic resonance imaging; OR, orientation reversal; $\mathrm{pFDR}, \mathrm{FDR}$ corrected $\mathrm{p}$-value; $\mathrm{T}_{\text {circ, }}^{2}$ circular $\mathrm{T}^{2}$-based test statistic; $\mathrm{SD}$, standard deviation; VEP, visual evoked potential; wPLI, weighted phase lag index. 


\section{Introduction}

Rapid developments in the neurological care of newborns and infants have raised the need to improve early neurological assessments for benchmarking early interventions as well as for individualizing therapeutic strategies (Spittle and Treyvaud 2016). Current evidence suggests that early therapeutic interventions may work best if they are started already during the first months after the birth (Spittle et al. 2015; Spittle and Treyvaud 2016). Such practice would need more sophisticated methods suitable for assessing the brain function in early infancy.

A range of clinical studies have shown that brain functions related to the infant's higher order visual information processing are involved in deficits found in many neurodevelopmental disorders (BrémondGignac et al. 2011; Stjerna et al. 2015), while visual system disorders are also a common mechanism underlying later neurocognitive compromise (Chorna et al. 2017; Leung et al. 2018). Hence, developing principled means for functional assessment of infant visual system could provide insight to a common pathway that links the medical adversities to neurodevelopmental compromise, i.e. valuable proxy tools for the assessment of wider neurocognitive development or its risks.

An established way for studying infant visual system is the assessment of cortical responses during repeated patterned stimulations. Three different stimuli, orientation reversal (OR), global form (GF), and global motion (GM), have been developed to specifically target putatively complementary pathways of cortical processing in the visual system (Wattam-Bell et al. 2010; Braddick and Atkinson 2011). In the infant context, cortical responses to global form and motion stimuli are especially interesting as they are suggested to employ ventral and dorsal visual stream mechanisms, respectively (Braddick et al. 2000; Braddick and Atkinson 2011), the two distinctly affected visual pathways after newborn adversities (Birtles et al. 2007; Leung et al. 2018). We have shown that this method, when coupled to eye tracking assistance (Ahtola et al. 2017), may offer reliable visual system studies at a few months of age, i.e. within the time frame with pressing clinical needs for new diagnostic options (Spittle et al. 2015; Spittle and Treyvaud 2016; Chorna et al. 2017).

Recent methodological developments in network neuroscience have shown promise for disclosing mechanisms that underlie various brain disorders (Bassett and Bullmore 2009; Bassett and Sporns 2017; Wig 2017), including brain effects after neonatal adversities (Tokariev et al. 2019a, 2019b). Those analyses are usually based on measuring large-scale correlations in measures of brain activity or blood flow during spontaneous brain activity, i.e. without apparent experimental tasks.

In addition to such networks of spontaneous neuronal activity, it is also possible to examine large-scale brain networks recruited in a more controlled manner using sensory stimulation, which has been successfully implemented in studies on cognitive brain mechanisms (e.g. working memory; Siebenhühner et al. 2016; Rodriguez-Larios and Alaerts 2019). In the context of primary sensory systems, use of periodic stimulation evokes steady-state electroencephalography (EEG) responses that are well characterized for the visual (Zhang et al. 2013; Norcia et al. 2015; Guo et al. 2018), somatosensory (Colon et al. 2012; Vakorin et al. 2019), and auditory systems (Picton et al. 2003; Farahani et al. 2017). They are used, for instance, in developing methods for brain-computer interface (BCl; Baek et al. 2019). The steady-state responses are typically studied as frequency-specific response at the given electrode, or cortical location only. Although this provides only a limited perspective to the investigated brain function that is likely to involve multiple brain regions via integrative oscillatory interactions (Bassett and Sporns 2017), there exist only a few prior works on exploring neuronal networks in the context of steady-state responses (e.g. Omidvarnia et al. 2014; Ying et al. 2015; Lea-Carnall et al. 2016; Vakorin et al. 2019).

Here, we set out to study whether cortical networks associated with steady-state responses after patterned visual stimuli (OR, GF, GM; Ahtola et al. 2017) in infants are as distinct as previously postulated (Braddick et al. 2000, 2005; Wattam-Bell et al. 2010), and if so, whether they could offer means for differential 
assessment of visual processing streams. After first characterizing the cortical networks evoked by OR/GF/GM stimuli in the cohort of typically developing 5-month-old infants, we also wanted to assess whether these network properties could potentially offer prediction of long-term outcomes by assessing their correlation with cognitive performance at two years of age.

\section{Materials and methods}

\subsection{Participants}

A cohort of typically developing infants was recruited ( $N=26 ; 9$ females). The infants were all born at fullterm ( $>37$ weeks of gestation) at the Helsinki University Hospital, and they had no medical issues as per parents' interviews. The recordings of the visual responses were performed at about five months of age (average age of $5.1 \pm 0.4$ months (SD)). Eighteen of these infants (see Supplementary Information S1) attended neuropsychological follow-up assessment at the mean age of $24.5 \pm 0.4$ months (SD) using Bayley III (Bayley 2006) that examines different aspects of infant's developmental status.

The Ethics Committee of Children's hospital in Helsinki University Hospital had approved the study protocol. The parents or guardians of the infants signed an informed consent to approve the involvement in this study. The individual shown in Figure $1 \mathrm{~A}$ has given a written informed consent to publish the photograph.

\subsection{Recording sessions}

An EEG cap was laid on infant's head and the infant was placed on caregiver's lap in front of the stimulation screen at viewing distance of $59 \pm 4 \mathrm{~cm}$ (SD; see the demonstration video at https://youtu.be/v8srf2Wwi2l (Vanhatalo 2014)). The visual stimuli were presented using Tobii T120 remote eye tracker (Tobii Technology $A B$, Stockholm, Sweden) that encompasses a built-in 17" thin-film transistor monitor (refresh rate $=60 \mathrm{~Hz}$, response time $=4 \mathrm{~ms}$ ). The eye tracking data, sampled at $120 \mathrm{~Hz}$ by the eye tracker, was used to guide the stimulation procedure online, and later to exclude invalid recording segments with gaze off-screen (setup described in detail in Ahtola et al. 2017). The brain responses to the stimulation were measured using a 31channel Waveguard EEG cap and 88-channel eego sports EEG amplifier (ANT-Neuro, Enschede, The Netherlands). The electrodes included were Fp1, Fp2, F7, F3, Fz, F4, F8, FC5, FC1, FC2, FC6, M1, T7, C3, Cz, $\mathrm{C} 4, \mathrm{~T} 8, \mathrm{M} 2, \mathrm{CP} 5, \mathrm{CP} 1, \mathrm{CP} 2, \mathrm{CP} 6, \mathrm{P} 7, \mathrm{P3}, \mathrm{Pz}, \mathrm{P} 4, \mathrm{P} 8, \mathrm{POz}, \mathrm{O} 1, \mathrm{Oz}, \mathrm{O} 2$ positioned consistently with the common the 10-10-system (see layout in Fig. 1C). The EEG caps had recording reference in $\mathrm{CPz}$, and we used sampling rate of $512 \mathrm{~Hz}$. As to stimulation, we targeted at least $100 \mathrm{~s}$ of data with appropriate gaze orientation per stimulus type.

\subsection{Visual stimuli}

We used three different, previously published visual stimuli: orientation reversal (OR), global form (GF), and global motion (GM). They were presented at the eye tracker screen as digital videos created in advance using the MATLAB functions of Psychophysics Toolbox (version 3; Brainard 1997). The video files are available upon request from the author.

Orientation reversal (OR). In the OR stimulus, black and white sine wave grating patterns (spatial resolution $0.45 \mathrm{cycle} /$ degree) alternate orientation between angles of $45^{\circ}$ and $135^{\circ}$ at reversal rate of $4 \mathrm{~Hz}$. On top of that, the phase of the grating pattern is set to change randomly at a higher, $24 \mathrm{~Hz}$ frequency. Because the local contrast variations in the stimulus are similar at every phase and orientation shift, this technique allowed us to separate the components elicited by the local changes from the orientation-specific response at the frequency domain. (Fig. 1B; Braddick et al. 2005).

Global form (GF). In GF stimulus, an ensemble of 2000 white arc segments (length $1.3^{\circ}$ ) is presented over a black background. The general alignment of the arcs alternate between coherent and non-coherent phases 
at $2 \mathrm{~Hz}$ rate (for full cycles). In the former phase, the arcs are arranged concentrically so that they create a perception of a global circular form pattern; In the latter phase, the orientations are randomized to lose the global form perception (Fig. 1B; Wattam-Bell et al. 2010).

Global motion (GM). In GM stimulus, an ensemble of 2000 white dots (diameter $0.3^{\circ}$ ) is presented over a black background, and their motion pattern switches at a rate of $2 \mathrm{~Hz}$ between two alternative phases. The coherent phase is perceived as global rotational movement where the dots move along mutually coherent, circular trajectories. In the non-coherent phase, the dots are moving along random and mutually unrelated trajectories (Fig. 1B; Wattam-Bell et al. 2010).

\subsection{Neuropsychological assessment}

Part of the original cohort $(\mathrm{N}=18)$ participated in a neuropsychological assessment at the age of two years. The assessment was performed according to Bayley Scales III (BSID-III; Bayley 2006) which is widely used in research and clinical context when assessing infants' neurodevelopment. BSID-III consists of five different subscales: cognitive, fine motor, gross motor, receptive language and expressive language subscales. For the present work, we selected the cognitive subscale from BSID-III as we aimed to examine relationships between the metrics of brain networks and general cognitive development. The cognitive scale tasks recruit several aspects of the cognitive performance such as visual perceptual abilities, symbolic play and object manipulation, memory, and early development of the conceptual reasoning and categorizing. The overall performance may be affected by the co-operation and self-regulation skills as well as the capability to comprehend given instructions.

\subsection{Signal analysis}

The overview of the EEG analysis pipeline is presented as a workflow schematic in Fig. 1C, and with more technical details in the Supplementary Figure S1 (see also Tokariev et al. 2019b).

\subsubsection{Pre-processing}

Raw EEG data was band-pass filtered around the stimulation frequency $(f=4 \mathrm{~Hz}$ for OR; $f=2 \mathrm{~Hz}$ for GF/GM) with a pair of digital low-pass and high-pass FIR filters with corresponding cut-off frequencies $(f \pm 0.3 \mathrm{~Hz}$, Hamming-window based linear-phase filter, order 1250, i.e. 5 cycles at $2 \mathrm{~Hz}$ ). To avoid phase shifts, we applied the filters both in forward and in backward directions. To reduce the computational load, the filtered data was then resampled down to $100 \mathrm{~Hz}$ (anti-aliasing filter applied).

Based on the eye tracking information, we selected the best 60 1-s-long epochs from each stimulation paradigm. This was done to minimize potential confound from differences between infants' performance across the tests. The accepted EEG epochs were concatenated to a single time series, and epochs with abnormally high peak-to-peak amplitude were excluded as plausible artefacts.

The epochs were selected based on gaze quality index which measured how much the infant had been watching the visual stimulus during a given time period. An optimization algorithm aimed to maximize this index in the epoch selection. Technical details of the gaze quality index calculation and the epoch selection procedure are described in the Supplementary Information S2. In addition, the MATLAB code for the optimization algorithm is shared as a downloadable package in https://github.com/ahtolee/GazeBasedEpochSelection together with test data from one recording.

Overall, the study setting allowed collection of data with very high performance; the average gaze quality index over the analysed time interval was $86 \%, 98 \%$, and $96 \%$ for OR, GF, and GM stimuli, respectively. A gaze quality index below $35 \%$ was taken as threshold for rejecting all data from the given condition in the given infant (two recordings were excluded with this criterion). Additionally, we also excluded the 
recordings where the total number of the artefact-free epochs was less than 60 (three recordings were excluded with this criterion).

\subsubsection{Computation of cortical signals}

To compute cortical source signals, we used a generic infant head model for all subjects. The model included scalp, skull, and intracranial volume surfaces that were derived from anatomical magnetic resonance imaging (MRI) data and approximated with 2562 equidistant vertices each (Tokariev et al. 2016). It is noteworthy that we used realistic conductivity values for these tissue types of infant head: $0.43 \mathrm{~S} / \mathrm{m}$ for scalp, $0.2 \mathrm{~S} / \mathrm{m}$ for skull, and $1.79 \mathrm{~S} / \mathrm{m}$ for cerebrospinal fluid (Despotovic et al. 2013; Odabaee et al. 2014). As a source space we opted to use 'Colin 27' cortical surface, available in Brainstorm software (Tadel et al. 2011), which was first appropriately scaled and smoothed to fit size and gyration of infant brain (Tokariev et al. 2019b).

Source space comprised 8014 dipoles of fixed normal orientation relative to cortical surface. Electrode positions in the head model corresponded to the EEG cap layout used at recordings (Fig. 1C). Forward solution was computed with symmetric boundary element method (Gramfort et al. 2010), and to get inverse solution, we applied dynamic statistical parametric mapping (Dale et al. 2000). Next, all 8014 cortical dipoles were collapsed into 58 parcels (Fig. 1C) and corresponding parcel signals were computed as weighted mean activity of sources within them (more details in Tokariev et al. 2019b). Finally, we labelled all parcels according to their anatomical location into frontal $(F)$, central $(C)$, temporal $(T)$, and occipital $(O)$ groups (Fig. 1C).

\subsection{3 $\mathrm{T}^{2}$ circ response detection}

We used circular $\mathrm{T}^{2}$ circ test statistic (Victor and Mast 1991) to determine whether the recorded EEG contained an evoked steady-state response. To this end, we applied an established paradigm (Wattam-Bell et al. 2010; Ahtola et al. 2017) that served as an additional step of quality control: all sessions without a statistically significant $(p<0.05)$ response in the postcentral parcel signals were excluded from the further network analyses (one session was excluded with this criterion). The family-wise error rate of the detections was controlled with the false discovery rate (FDR) correction by Benjamini and Hochberg (1995) with q-value threshold set to 0.05 .

After the exclusion procedures, the final number of the accepted recordings per stimulus type were 23, 25, and 24 for OR, GF, and GM, respectively. In the further discussion, this dataset is referred as the full dataset. Some of our statistical analyses were performed pairwise between stimulus types, therefore needing data from all three conditions that was available from 22 infants. Correspondingly, this dataset is referred as the pairwise dataset. For individual break out of these results, see Supplementary Information S1.

\subsubsection{Calculation of functional connectivity}

Functional connectivity between pairs of cortical parcel signals $(58 \times 58)$ was estimated using debiased estimator of the squared weighted phase lag index (wPLI; Vinck et al. 2011), which aims to minimize potential confounding by linear mixing of common sources that are typically seen at near zero phase lags. The values of WPLI range from 0 and 1, referring to completely uncorrelated and fully synchronized signals, respectively. The resulting connectivity matrix is then taken as functional network where parcels are nodes and the corresponding WPLI values are edges representing strength of functional interaction between them (see example in Fig. 1C).

There is a certain level of variation on how reliably the measured (and transformed) signals can describe the connectivity between the parcels in the current recording configuration. In order to remove spurious interactions that may arise from compromised reliability in our model, we used a binary fidelity operator that rejects unreliable connections (for more details, see Tokariev et al. 2019b). With our recording 
constellation, this procedure rejected $20 \%$ of all possible edges from further analysis, leaving 1330 edges to the final network.

\subsubsection{Analysis of response networks}

Consistent networks. Starting from the individual adjacency matrices, we calculated consistent networks (CN), i.e. networks that comprise the strongest edges most consistently across the cohort (Tokariev et al. 2019b). The calculations were done at group-level separately for OR, GF, and GM stimulus. In short, we first determine the strongest $20 \%$ edges in each adjacency matrix (10\% and $30 \%$ were also tested for control). The resulting binary arrays were combined per stimulus condition over the cohort to yield a matrix with only strongest edges per stimulus condition. A binomial test was used to assess consistency of edges across the cohort, setting probability of success to $20 \%$, alpha level to 0.05 , and trial number to the number of infants. Multiple comparisons were controlled by rejecting a fraction of the weakest significant observations, where $5 \%$ fraction was taken from the overall number of tests to correspond to the expected level of false positives (Palva et al. 2010). Next, the network density was calculated as a fraction between the number of consistent edges and all possible network edges. Network densities were computed both globally and regionally by calculating the proportion of edges that belong to a specific area (frontal, central, temporal, occipital) and were included in the CN. Generally, higher density would indicate a wider network that entrains larger number of nodes. Finally, we calculated average strengths of the consistent networks (CN wPLI), taken as the mean wPLI value of the edges belonging to the given $\mathrm{CN}$. Here, the $\mathrm{CN}$ derived at group-level was used as the mask for computing mean wPLI for each infant separately. These CN wPLI values were used in a correlation analysis aimed to examine the associations between the metrics of brain networks and cognitive development.

Graph measures. In addition to the regional comparisons, we also studied networks using two graph theoretical metrics that are taken to quantify different aspects of functional segregation and integration of a complex network: clustering coefficient (CC) and characteristic path length (CPL) (Rubinov and Sporns 2010). The optimal balance between these two coexisting properties is essential for the processes in largescale human brain networks. In general, segregation gives brain the ability for specialized processing to occur within functionally isolated subsystems, whereas integration provides a way for associating interactions across the distinct modules (Wig 2017).

Statistics. The statistical comparisons between the networks related to the stimuli were done using pairwise Wilcoxon signed-rank test, or using nonparametric Kruskall-Wallis when all three stimuli were compared. Alpha level for both tests was set to 0.05 with FDR correction applied to control multiple comparisons (Benjamini and Hochberg 1995; q-level set to 0.20 unless stated otherwise). For the grouplevel comparisons of the graph measures, we used nonparametric Wilcoxon rank-sum test. The correlations between average strengths of the consistent networks and cognitive scores from BSID-III were calculated using Pearson's method.

\subsection{Analysis software}

All calculations and signal processing steps were done with custom-scripted MATLAB routines (version R2018A, MathWorks, Natick, MA, USA) and freely available toolboxes. A head model was computed using openMEEG Toolbox (Gramfort et al. 2010). Brainstorm Toolbox (Tadel et al. 2011) was used to compute cortical source signals. Brain Connectivity Toolbox (Rubinov and Sporns 2010) provided the functions for calculations of the network metrics. Code for computation of consistent networks is freely available online from https://github.com/babyEEG/neoNets. The full analysis pipeline is available from the corresponding authors upon request. 


\section{Results}

\subsection{Cortical responses to patterned stimuli are reliably detected in infants}

Analysis of $\mathrm{T}^{2}$ circ responses in each cortical parcel showed that apart from one OR recording, every infant yielded significant postcentral responses for all three stimuli. Projecting the mean $\mathrm{T}^{2}$ circ results on a topographical 3D model (Fig. 2) shows that all stimuli resulted in responses mainly on the occipital area. The GM responses, however, also extended wider towards temporal and central areas, which is clearly noticeable also in the corresponding parcel-specific average response rates that are presented in the Supplementary Figure S2.

\subsection{Cortical networks are modified by the type of visual stimulus}

Functional networks were further examined visually using circular connectivity diagrams (Fig. 3A; full dataset) and consistent network graphs (Fig. 3C; full dataset), as well as statistically though comparison of averaged regional WPLI values (Fig. 3B; pairwise dataset). The consistent networks were calculated from the strongest $20 \%$ edges in the individual networks, but corresponding results with $10 \%$ and $30 \%$ are presented the Supplementary Figure S3.

The network related to OR stimulus was mainly occipital, including some temporal areas (Fig. 3A), and only very few frontal or central connections (Fig. 3C). The strongest edges in the GF responses provided a pattern similar to OR, however GF connections were generally weaker. The CN associated with GF stimulus extended far more to frontal and central regions, as also seen in the highest global density value of all three stimuli (5.2\%; Fig. 3C). GM stimulus, in turn, resulted in the strongest global network, recruiting large areas across the cortex. Compared to the GF stimulus, the $\mathrm{CN}$ related to the GM stimulus was more uniformly spread across the regions (Fig. 3C).

Statistical comparison (Kruskall-Wallis) between all three stimuli showed significant regional differences only at connections that involve frontal areas (Fig. 3B). This was most probably due to the generally weak frontal connections in the OR network. All the aforementioned findings sustained the FDR correction for multiple comparisons (Benjamini and Hochberg 1995; q-level 0.20). The post hoc analysis, performed between pairs of stimulus types showed most differences between OR and GM (Fig. 3B), and all differences involved frontal areas (F, FF, FC, FO). Some differences were also seen between OR and GF, especially in connections involving frontal areas (GF stronger; F, FF), but these findings did not survive the FDR correction.

Since the visual inspection of connectivity graphs suggested rather global differences, we next assessed whether global graph measures would be able to differentiate between stimulus conditions. To this end, we used metrics that are often taken to reflect the level of functional integration and segregation, clustering coefficient (CC), characteristic path length (CPL), respectively. CC was computed for each node separately, hence allowing topographic assessment as well (Fig. 4A; full dataset). Comparison of the group mean CC distributions indicated that the topographic CC patterns of OR and GF responses are rather comparable, with tightly connected clusters mainly in the occipital and temporal areas. However, GM appeared to be different, with typically high average CC values throughout all cortical regions, which was also confirmed in the statistical comparison of CC levels in different regions (Fig. 4B; full dataset). In addition, central, temporal, and occipital regions showed significant differences between GF and GM responses, whereas GM and OR networks were different in the frontal region. Analysis of global CPL values (Fig. 4C, full dataset) showed that GM networks are typically the shortest (low CPL) and they are statistically significantly different from both OR and GF. However, CPL in networks related to OR and GF are comparable. Comparison of average wPLI strengths within the consistent networks (CN wPLI) showed that GF stimulus leads to generally weak wPLI, which is significantly weaker than the network seen after GM stimulus (Fig. 4C, full dataset). 


\subsection{Networks related to global form processing correlate with later neurocognition}

As the cortical networks appeared to differ between stimulus modalities, we then wanted to assess whether these networks associated with infant cortical visual processing could also be associated with later neurocognitive development. To this end, the cognitive neuropsychological scores of the infants at two years of age were compared to the strength of networks using the group-level, stimulus-specific consistent networks as templates (CN wPLI; Fig. 5).

We found positive association ( $r=0.47 ; p=0.042$, Pearson; $p F D R=0.126$, Benjamini-Hochberg) between BSID-III cognitive scale scores and the strengths of GF consistent network. At the same time, no significant relationships were found between OR or GM networks versus later cognitive performance scores (both had $r<0.07$ and uncorrected $p>0.79$ ). Together these results perhaps indicate that GF stimulus might trigger specific network patterns that play important role for later cognitive processing.

\section{Discussion}

Our findings show that cortical source responses to complex visual stimuli can be reliably recorded in the infants, and the responses encompass brain-wide networks with at least partial specificity to the given stimulus condition. The observations are fully compatible with prior literature showing the responses in individual scalp EEG signals (Braddick et al. 2005; Wattam-Bell et al. 2010; Ahtola et al. 2017). We extend earlier knowledge by characterizing the cortico-cortical interactions and their differences between stimuli, which are considered to recruit distinct mechanisms in the visual system (Braddick and Atkinson 2011). By analysing brain responses from the transformed source signals instead of scalp EEG derivations, we obtained response rates that were even higher than previously reported, yielding response detections in $96-100 \%$ of infants.

The nodal level response, $\mathrm{T}^{2}$ circ, was predominantly found in the occipital area, which is around primary visual area and hence expected as the first relay for cortical visual processing of all stimulus types. Yet, we found significant response components also outside the occipital lobe, supporting the idea of wider cortical involvement in visual information processing, such as parietal, temporal and frontal areas. While this was expected based on prior scalp level analyses (Wattam-Bell et al. 2010; Ahtola et al. 2017), it also prompted the idea of looking for brain wide network interactions. Moreover, the conventional method for assessing responses locally from the $\mathrm{T}^{2}$ circ test statistic is technically efficient for binary assessment (Victor and Mast 1991), however it suffers from significant sensitivity to technical peculiarities such as varying amounts of data (Ahtola et al. 2017), calling for more sophisticated means to look for global brain responses.

Visual comparison of networks after different visual stimuli suggested that the OR network is locally strongest, but spatially constrained predominantly to the occipital regions, while the GM and GF responses encompass much wider distribution. Compared to GF, the connections within a typical GM related network are generally stronger and comprise a network structure that consist of multiple regional hubs that share strong local connectivity between the neighbouring nodes. The differences between modalities were also supported by statistical comparison of several network-derived measures. These observations are by and large compatible with the prior idea that OR stimulus is primarily processed in and near the primary visual areas. Our findings from the GM and GF responses are, however, partly challenging the perhaps simplistic view (e.g. Braddick and Atkinson 2011) that processing of GM and GF stimuli would be limited to dorsal and ventral streams, respectively). Indeed, our network findings provide no evidence for such distinction, while they rather speak for a brain-wide involvement. It is possible that such near-global recruitment is at least partly due to the immaturity of high order visual systems (Wattam-Bell et al. 2010; Braddick and Atkinson 2011), however recent literature using network neuroscience framework provides ample evidence for the idea that higher order processing would be rather more distributed than localized (van den Heuvel and Sporns 2013; Wig 2017; Hirvonen et al. 2018). 
With respect to conclusions on sensory physiology, our study has limitations that may arise from the analytic approach but also partly from unavoidable individual variability. Here we assessed phase synchronization at the stimulation frequency, which allows assessment of the networks related to the steady-state response. However, higher order cortical processing, at least in the older subjects, is shown to involve large-scale phase synchrony at higher frequencies. It is possible that our findings are more about cortical entrainment by the stimulus (Zhang et al. 2013), while analysis of e.g. alpha and beta frequency phase synchrony could disclose more delimited networks related to the perception. Such analysis requires substantial efforts to account for spurious findings; it would be useful to perform such analyses on datasets with more clinical variability to allow better searching for correlations with clinical performance at the same time.

Prior work has suggested that the steady-state VEPs could be potentially used for outcome assessment, or prediction of future neurocognitive development (Mercuri et al. 1998; Braddick and Atkinson 2011). Recent development of the setup using eye tracking control and optimized analysis tools yields method performance (e.g. sensitivity and specificity) that would allow its use in clinical diagnostics. Our present finding supports such idea by also showing different brain-wide networks associated with each stimulus type. Indeed, we found an interesting positive association between GF consistent networks and cognitive performance at two years of age. This finding should be considered only as preliminary observation that is promising when considering the relatively narrow range in the typically seen infant population. More data with much wider range of outcomes is needed to evaluate the true clinical reliability and/or added value in the context of other known information. Those studies would also need to benchmark the VEP networks with more fine-grained assessment of visual cognitive skills, to show, e.g. the potential for differential assessment of visual processing streams.

\section{Acknowledgements}

Our sincere thanks go to the technicians at BABA Center in Children's Hospital for conducting the measurements. This work was supported by the Helsinki University Hospital Funds [1C27012974], Academy of Finland [253130] and the TERVA program of Finnish Academy [314450], Neuroscience Center at University of Helsinki, Lastentautien Tutkimussäätiö, and Juselius Foundation. The sponsors were not involved in the collection, analysis and interpretation of data or in the writing of the manuscript.

\section{Conflict of interest}

None. 


\section{References}

Ahtola E, Stjerna S, Stevenson N, Vanhatalo S. Use of eye tracking improves the detection of evoked responses to complex visual stimuli during EEG in infants. Clin Neurophysiol Pract. 2017;2:81-90.

Baek HJ, Chang MH, Heo J, Park KS. Enhancing the Usability of Brain-Computer Interface Systems. Comput Intell Neurosci. 2019. https://doi.org/10.1155/2019/5427154. Article ID 5427154, 12 pages.

Bassett DS, Bullmore ET. Human Brain Networks in Health and Disease Danielle. Curr Opin Neurol. 2009;22:340-7.

Bassett DS, Sporns O. Network neuroscience. Nat Neurosci. 2017;20:353-64.

Bayley N. Bayley Scales of Infant and Toddler Development-Third Edition: Technical manual. San Antonio, TX: Harcourt Assessment; 2006.

Benjamini Y, Hochberg Y. Controlling The False Discovery Rate - A Practical And Powerful Approach To Multiple Testing. J R Stat Soc, Ser B. 1995;57:289-300.

Birtles DB, Braddick OJ, Wattam-Bell J, Wilkinson AR, Atkinson J. Orientation and motion-specific visual cortex responses in infants born preterm. Neuroreport. 2007;18:1975-9.

Braddick O, Atkinson J. Development of human visual function. Vision Res. 2011;51:1588-609.

Braddick O, Birtles D, Wattam-Bell J, Atkinson J. Motion- and orientation-specific cortical responses in infancy. Vision Res. 2005;45:3169-79.

Braddick OJ, O'Brien JMD, Wattam-Bell J, Atkinson J, Turner R. Form and motion coherence activate independent, but not dorsal/ventral segregated, networks in the human brain. Curr Biol. 2000;10:731-4.

Brainard DH. The Psychophysics Toolbox. Spat Vis. 1997;10:433-6.

Brémond-Gignac D, Copin H, Lapillonne A, Milazzo S, European Network of Study. Visual development in infants: physiological and pathological mechanisms. Curr Opin Ophthalmol. 2011;22 Suppl.

Chorna OD, Guzzetta A, Maitre NL. Vision Assessments and Interventions for Infants 0-2 Years at High Risk for Cerebral Palsy: A Systematic Review. Pediatr Neurol. 2017;76:3-13.

Colon E, Legrain V, Mouraux A. Steady-state evoked potentials to study the processing of tactile and nociceptive somatosensory input in the human brain. Neurophysiol Clin. 2012;42:315-23.

Dale AM, Liu AK, Fischl BR, Buckner RL, Belliveau JW, Lewine JD, et al. Dynamic statistical parametric mapping: combining $\mathrm{fMRI}$ and MEG for high-resolution imaging of cortical activity. Neuron. 2000;26:55-67.

Despotovic I, Cherian PJ, De Vos M, Hallez H, Deburchgraeve W, Govaert P, et al. Relationship of EEG sources of neonatal seizures to acute perinatal brain lesions seen on MRI: a pilot study. Hum Brain Mapp. 2013;34:2402-17.

Farahani ED, Goossens T, Wouters J, van Wieringen A. Spatiotemporal reconstruction of auditory steadystate responses to acoustic amplitude modulations: Potential sources beyond the auditory pathway. Neuroimage. 2017;148:240-53.

Gramfort A, Papadopoulo T, Olivi E, Clerc M. OpenMEEG: opensource software for quasistatic bioelectromagnetics. Biomed Eng Online. 2010;9:45.

Guo D, Guo F, Zhang Y, Li F, Xia Y, Xu P, et al. Periodic visual stimulation induces resting-state brain network reconfiguration. Front Comput Neurosci. 2018;12:1-11. 
van den Heuvel MP, Sporns O. Network hubs in the human brain. Trends Cogn Sci. 2013;17:683-96.

Hirvonen J, Monto S, Wang SH, Palva JM, Palva S. Dynamic large-scale network synchronization from perception to action. Netw Neurosci. 2018;2:442-63.

Lea-Carnall CA, Montemurro MA, Trujillo-Barreto NJ, Parkes LM, El-Deredy W. Cortical Resonance Frequencies Emerge from Network Size and Connectivity. PLoS Comput Biol. 2016;12:1-19.

Leung MPS, Thompson B, Black J, Dai S, Alsweiler JM. The effects of preterm birth on visual development. Clin Exp Optom. 2018;101:4-12.

Mercuri E, Braddick O, Atkinson J, Cowan F, Anker S, Andrew R, et al. Orientation-reversal and phasereversal visual evoked potentials in full-term infants with brain lesions: a longitudinal study. Neuropediatrics. 1998;29:169-74.

Norcia AM, Appelbaum LG, Ales JM, Cottereau BR, Rossion B. The steady-state visual evoked potential in vision research: A review. J Vis. 2015;15:4.

Odabaee M, Tokariev A, Layeghy S, Mesbah M, Colditz PB, Ramon C, et al. Neonatal EEG at scalp is focal and implies high skull conductivity in realistic neonatal head models. Neuroimage. 2014;96:73-80.

Omidvarnia A, Azemi G, Boashash B, Otoole JM, Colditz PB, Vanhatalo S. Measuring time-varying information flow in scalp EEG signals: Orthogonalized partial directed coherence. IEEE Trans Biomed Eng. 2014;61:680-93.

Palva JM, Monto S, Kulashekhar S, Palva S. Neuronal synchrony reveals working memory networks and predicts individual memory capacity. Proc Natl Acad Sci U S A. 2010;107:7580-5.

Picton TW, John MS, Dimitrijevic A, Purcell D. Human auditory steady-state responses. Int J Audiol. 2003;42:177-219.

Rodriguez-Larios J, Alaerts K. Tracking transient changes in the neural frequency architecture: harmonic relationships between theta and alpha peaks facilitate cognitive performance. J Neurosci. 2019;39:2919-18.

Rubinov M, Sporns O. Complex network measures of brain connectivity: uses and interpretations. Neuroimage. 2010;52:1059-69.

Siebenhühner F, Wang SH, Palva JM, Palva S. Cross-frequency synchronization connects networks of fast and slow oscillations during visual working memory maintenance. Elife. 2016;5:15-30.

Spittle A, Treyvaud K. The role of early developmental intervention to influence neurobehavioral outcomes of children born preterm. Semin Perinatol. 2016;40:542-8.

Spittle A, Orton J, Anderson PJ, Boyd R, Doyle LW. Early developmental intervention programmes provided post hospital discharge to prevent motor and cognitive impairments in preterm infants. Cochrane Database Syst Rev. 2015;(11):CD005495. doi: 10.1002/14651858.CD005495.pub4.

Stjerna S, Sairanen V, Gröhn R, Andersson S, Metsäranta M, Lano A, et al. Visual fixation in human newborns correlates with extensive white matter networks and predicts long-term neurocognitive development. J Neurosci. 2015;35:4824-9.

Tadel F, Baillet S, Mosher JC, Pantazis D, Leahy RM. Brainstorm: a user-friendly application for MEG/EEG analysis. Comput Intell Neurosci. 2011;2011:879716.

Tokariev A, Roberts JA, Zalesky A, Zhao X, Vanhatalo S, Breakspear M, et al. Large-scale brain modes reorganize between infant sleep states and carry prognostic information for preterms. Nat Commun. 2019a;10:2619. 
Tokariev A, Stjerna S, Lano A, Metsäranta M, Palva JM, Vanhatalo S. Preterm Birth Changes Networks of Newborn Cortical Activity. Cereb Cortex. 2019b;29:814-26.

Tokariev A, Vanhatalo S, Palva JM. Analysis of infant cortical synchrony is constrained by the number of recording electrodes and the recording montage. Clin Neurophysiol. 2016;127:310-23.

Vakorin VA, Ross B, Doesburg SM, Ribary U, Mclntosh AR. Dominant patterns of information flow in the propagation of the neuromagnetic somatosensory steady-state response. Front Neural Circuits. 2019;12:1-9.

Vanhatalo S. Eyetracking of babies BABAcenter. 2014. Available from: https://youtu.be/v8srf2Wwi2I

Victor JD, Mast J. A new statistic for steady-state evoked potentials. Electroencephalogr Clin Neurophysiol. 1991;78:378-88.

Vinck M, Oostenveld R, van Wingerden M, Battaglia F, Pennartz CMA. An improved index of phasesynchronization for electrophysiological data in the presence of volume-conduction, noise and sample-size bias. Neuroimage. 2011;55:1548-65.

Wattam-Bell J, Birtles D, Nyström P, von Hofsten C, Rosander K, Anker S, et al. Reorganization of Global Form and Motion Processing during Human Visual Development. Curr Biol. 2010;20:411-5.

Wig GS. Segregated Systems of Human Brain Networks. Trends Cogn Sci. 2017;21:981-96.

Ying J, Zhou D, Lin K, Gao X. Network analysis of functional brain connectivity driven by gamma-band auditory steady-state response in auditory hallucinations. J Med Biol Eng. 2015;35:45-51.

Zhang Y, Xu P, Huang Y, Cheng K, Yao D. SSVEP Response Is Related to Functional Brain Network Topology Entrained by the Flickering Stimulus. PLoS One. 2013;8:e72654. 


\section{Figure captions}

\section{Fig. 1. Study design and analysis workflow.}

A) During the recording, infant is placed on caregiver's lap with EEG cap in place, and visual stimulations are presented from the screen of the eye tracker. B) Still images of the two alternating phases of each visual stimulation (OR, orientation reversal; GF, global form; GM, global motion). The white square in the lower right corner was used for delivering timing impulse to the EEG system to ensure synchronization. C) An illustration of the EEG analysis pipeline. The filtered EEG segments were selected based on the gaze data and transformed into cortical parcel signals. Then analytic phase was extracted to allow computation of phase-phase interactions. The resulting functional networks were assessed using nodal and global network metrics, and the results were visualized over 3D cortex model or circular graph.

\section{Fig. 2. Spatial distribution of the $\mathrm{T}^{2}$ circ responses.}

$\mathrm{T}^{2}$ circ statistic was used to detect the presence of statistically significant response at the parcel level. Figures depict spatial distributions of the mean $\mathrm{T}^{2}$ circ values over the study cohort (full dataset) projected on a 3D cortex model. Results for the three stimuli are presented separately (OR, orientation reversal; GF, global form; GM, global motion). In the figures, each dot represents centroid of a parcel, coloured according to its $\mathrm{T}^{2}$ circ value. Note the GM response components also outside the occipital area.

\section{Fig. 3. VEP response networks.}

A) Circle diagrams visualize the mean wPLI over all infants in the given edges (strongest 20\%). Note the dominance of posterior connections in the orientation reversal (OR) network, while global form (GF) and motion (GM) connections are more uniformly distributed. B) Comparison of regional ( $F$, frontal; $C$, central; $\mathrm{T}$, temporal; $\mathrm{O}$, occipital) connection strengths shows that OR differs most from the other modalities. The leftmost block between vertical separators contains intra-regional intra-hemispheric connections, the second block contains intra-regional inter-hemispheric connections, and the third block inter-regional intra-hemispheric connections. Additionally, the three columns labelled 'All' contain all connections of that block type. Each box is coloured according to group-level mean wPLI of the corresponding subset of connections. An asterisk marker $\left({ }^{*}\right)$ above a column indicates that the differences between the three networks were statistically significant ( $p<0.05 ;$ Kruskall-Wallis). A vertical segment between two rows indicates that the differences between the two conditions was statistically significant in a post-hoc test $(p<0.05$; Wilcoxon signed-rank). The findings coloured with grey yielded significant $p$-values but failed in the subsequent FDR correction. C) Circle diagrams show the $\mathrm{CN}$ of each stimulus condition, and the colour hues reflect the frequency (prevalence) of the given edge in the cohort. The bars below depict global $(G)$ and regional $(F, C, T, O)$ densities of the $C N$ s. The $3 D$ cortex models in the bottom visualize the given $C N s$ for easier topographical assessment (same as in the circle graph above). Note the overall difference where OR is mostly occipital while GM and GF relate to a more global CN.

\section{Fig. 4. Network metrics.}

A) The figures depict the group-level topographies of the clustering coefficients (CC) of the VEP networks (averaged over the cohort). Results for the three stimuli are presented separately (OR, orientation reversal; GF, global form; GM, global motion). B) The figure shows distributions of the spatially averaged CC values for each infant. In the regional breakdown, the clustering coefficients in parcels were grouped based on the brain regions they belong to. The 'Global' condition covers all regions. Each violin graph depicts the median value (horizontal line), the probability density estimate of the inspected distribution (vertical envelope), and the actual data points. Statistically significant ( $p<0.05$; Wilcoxon rank-sum) differences between the stimulus types are marked with asterisk signs. C) The figure shows violin plot distributions of characteristic path length (left) and mean wPLI in the edges belonging to a consistent network (CN wPLI; right) for each infant. An asterisk marker denotes a statistically significant ( $p<0.05$; Wilcoxon rank-sum) differences in a comparison between two stimuli. 
Fig. 5. Correlation of the network strength with cognitive outcome at 2 years of age.

The graphs show relationship of VEP network strengths (CN wPLI, mean wPLI in the edges of a consistent network) at 5 months and the cognitive scores at 2 years of age (least-squares fit). The $r$ denotes correlation coefficients, whereas $p$ and pFDR denote significance levels of the correlation coefficients before and after false discovery rate correction (Benjamini-Hochberg), respectively.

\section{Supplementary material}

\section{Supplementary Information S1. Measurement information table.}

The table shows information of the measurements and effect of the epoch segmentation for all infants within the studied cohort (\#1-\#26). Data is presented separately for all three stimulus types (OR, orientation reversal; GF, global form; GM, global motion). The first row shows whether an adequate amount of artefact-free EEG was available for the analysis ( $x=y e s)$. The first row shows the general gaze quality within the 60 epochs that were selected for the response analysis. Measurements with gaze quality less than $35 \%$ were excluded from the further analyses (marked with red). The third row shows whether a statistically significant ( $p<0.05 ; \mathrm{T}^{2}$ circ) posterior response could be detected from the analysed data ( $x=$ yes). Recordings without a response were excluded from the further analyses.

The column on the right shows the number of valid recordings along the described exclusion steps. From the cohort of 26 infants, we could finally analyse $23 \mathrm{OR}, 25 \mathrm{GF}$, and $24 \mathrm{GM}$ recordings (full dataset). The average gaze qualities of these recordings are given in the same column ('AVG'). The number of infants with valid data from all three stimulus types was 22 (pairwise dataset).

Part of the original cohort $(\mathrm{N}=18$ ) participated also in a neuropsychological assessment (Bayley Scales III) at the age of two years. The last row presents their cognitive subscale scores that were used in the correlation analysis. Labels of the infants with adequate VEP tests, but missing cognitive scores are marked with orange.

\section{Supplementary Information S2. Use of the eye tracker data and gaze-based epoch selection algorithm.} Illustrated section describes in detail how the eye tracking data was used in this study. This includes descriptions of the data structures, calculation of the gaze quality index, and design of the gaze data -based epoch selection algorithm that aim to maximize the gaze quality within the analysed epochs.

\section{Supplementary Figure S1. Workflow of the test paradigm.}

The flow chart compiles the signal processing pipelines that were used in the work, starting from the data acquisition, and resulting in the network metrics and statistical analyses. Boxes show all the major processing steps and the specific parameters for them are given in brackets.

Supplementary Figure S2. $\mathrm{T}^{2}$ circ response rates.

We used $\mathrm{T}^{2}$ circ statistic to evaluate of the statistical significance of an evoked steady-state responses, and applied it as a criterion for the recordings to be included in the subsequent connectivity analyses. The figures here depict the group-level topography of the nodal average response rates $(p<0.05)$ for all three stimulus types (OR, orientation reversal; GF, global form; GM, global motion).

\section{Supplementary Figure S3. Effect of the alleged network size on the consistent network analysis.}

Consistent network (CN) comprise the set of the strongest edges that emerge coherently across the cohort. The calculation of $\mathrm{CN}$ started by determination of certain amount of strongest edges in each adjacency matrix. This amount $(k)$ specifies the alleged size of the network of which consistency is being inspected. We wanted to see how the $\mathrm{CN}$ properties would change, if different $k$-values were used. The 
Supplementary Figures S3 presents the consistent network analyses similar to Fig. $3 C$, for $k=10 \%, 20 \%$, and $30 \%$. Note how GF network gets denser and GM network sparser, when $k$ is increased. 
A

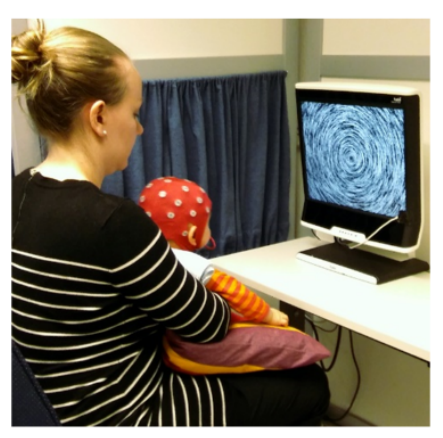

B

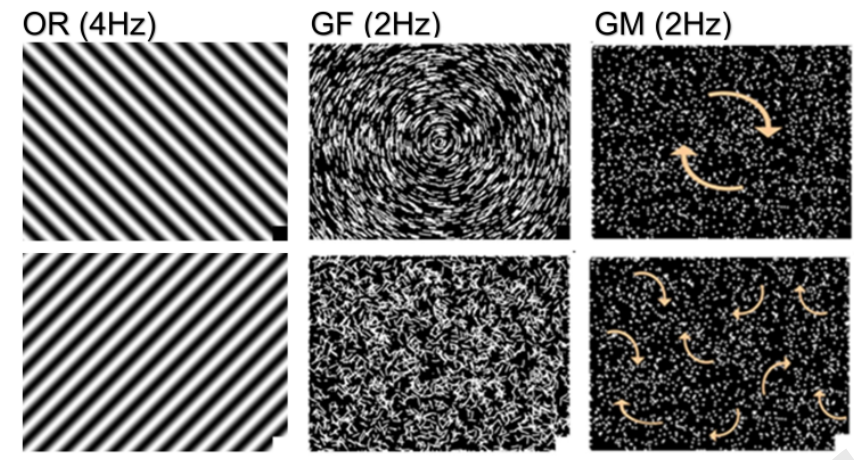

C
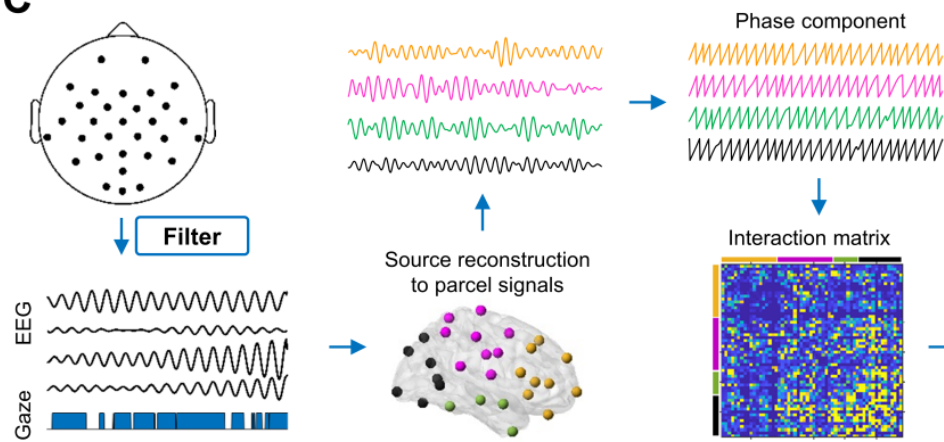

Source reconstruction
to parcel signals
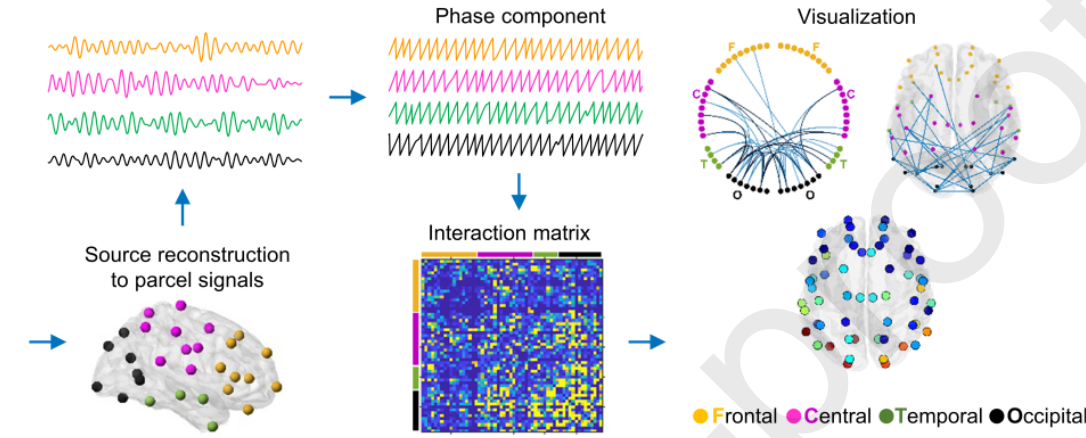

OR

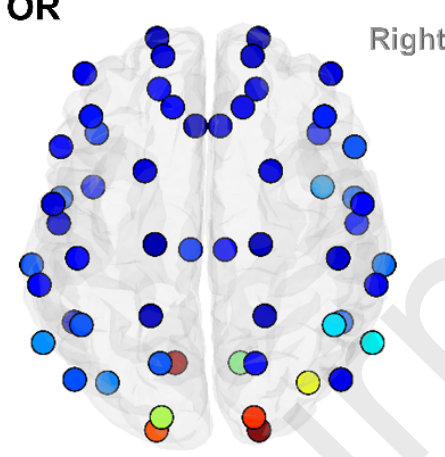

GF

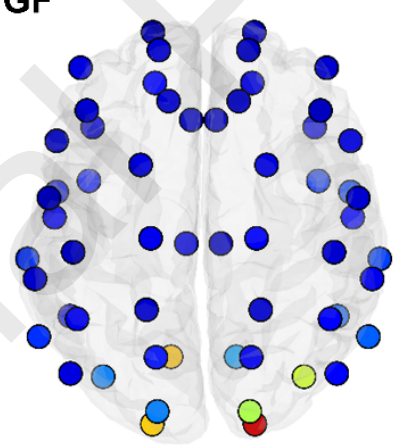

GM

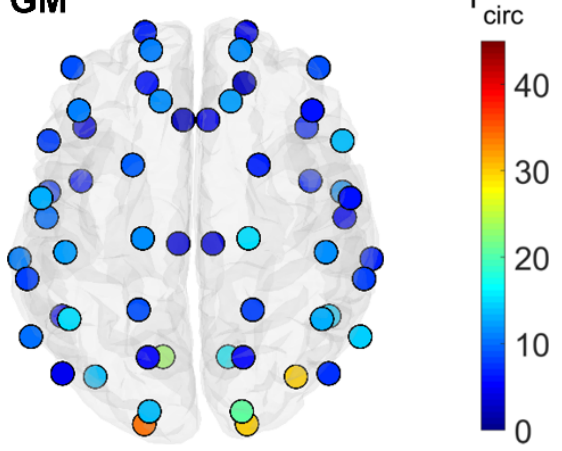


A Strongest networks OR

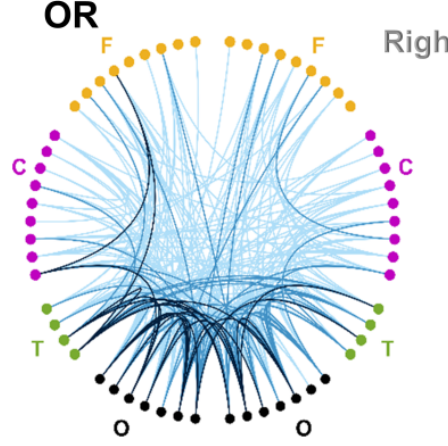

GF

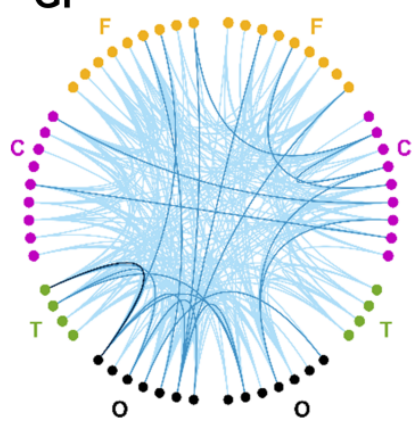

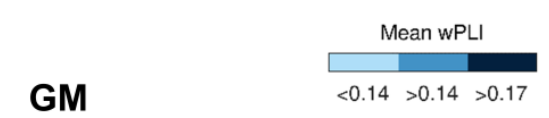

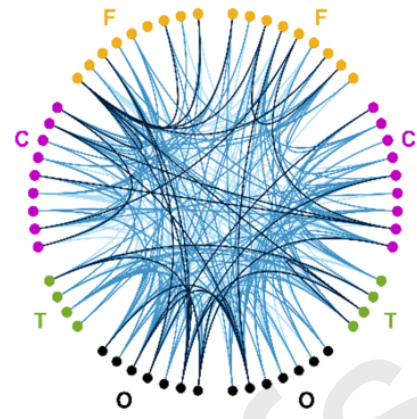

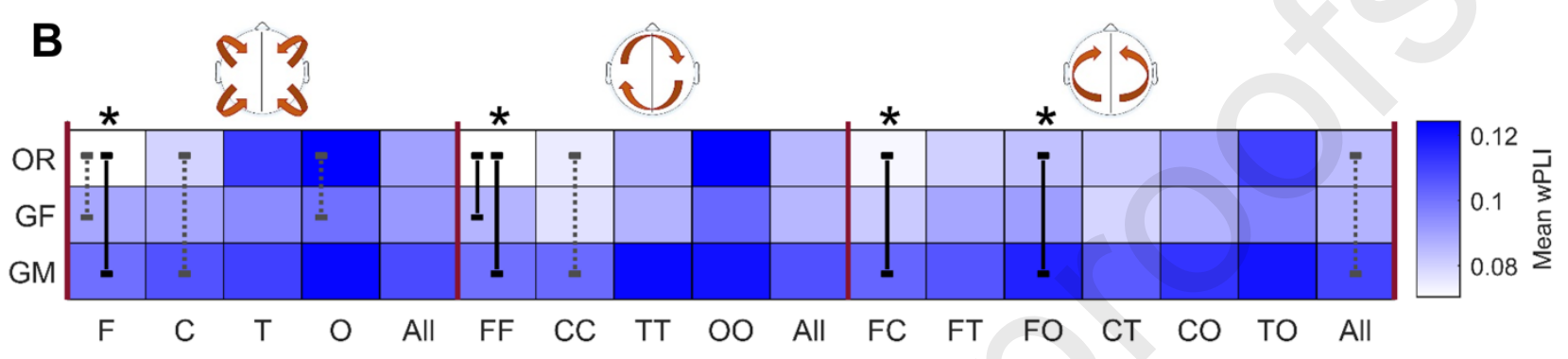

C Consistent networks
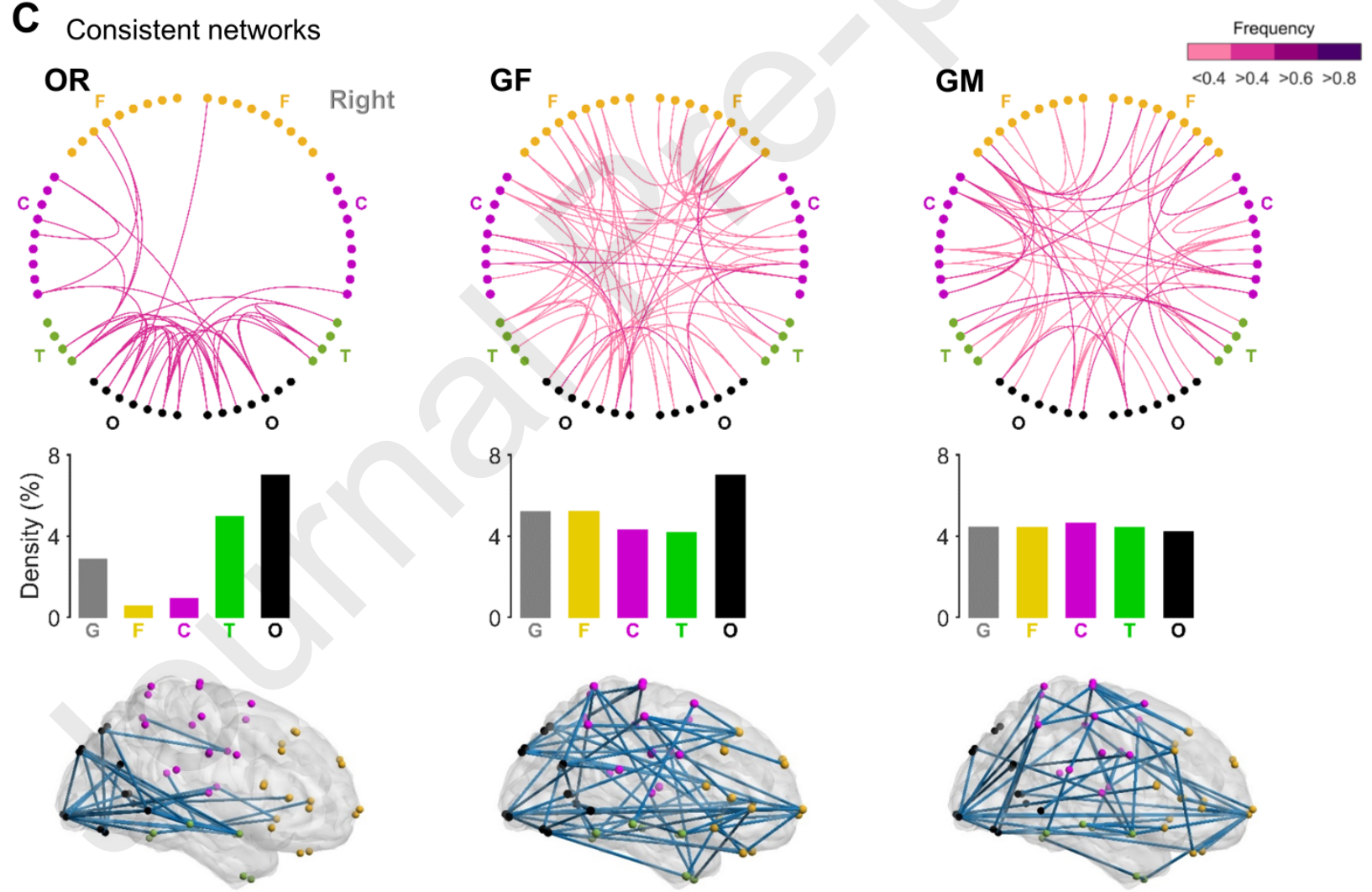

G Global, F Frontal, C Central, T Temporal, O Occipital 
A OR
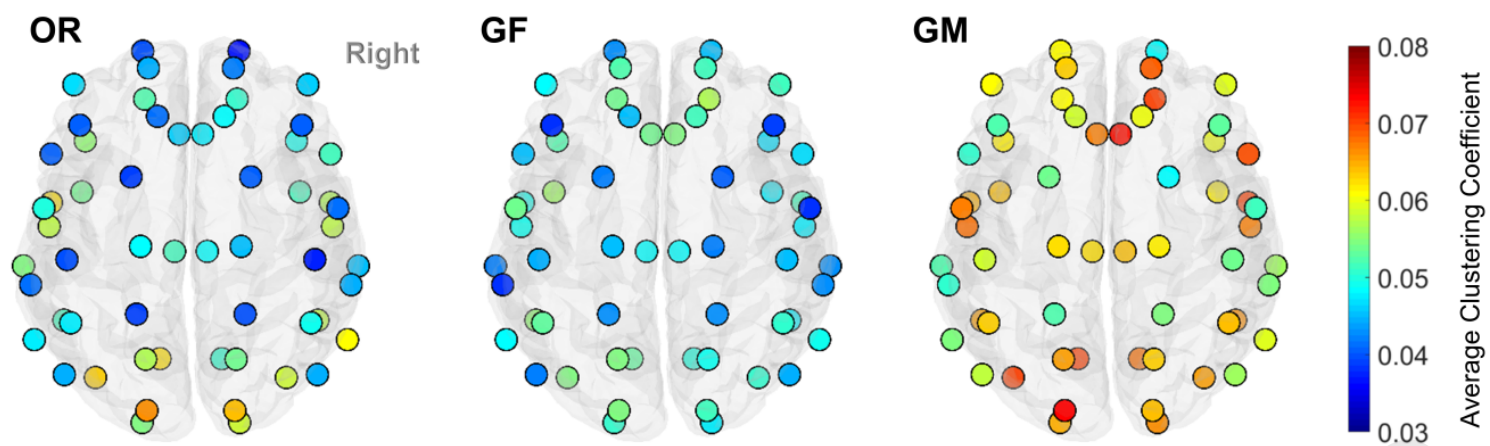

B

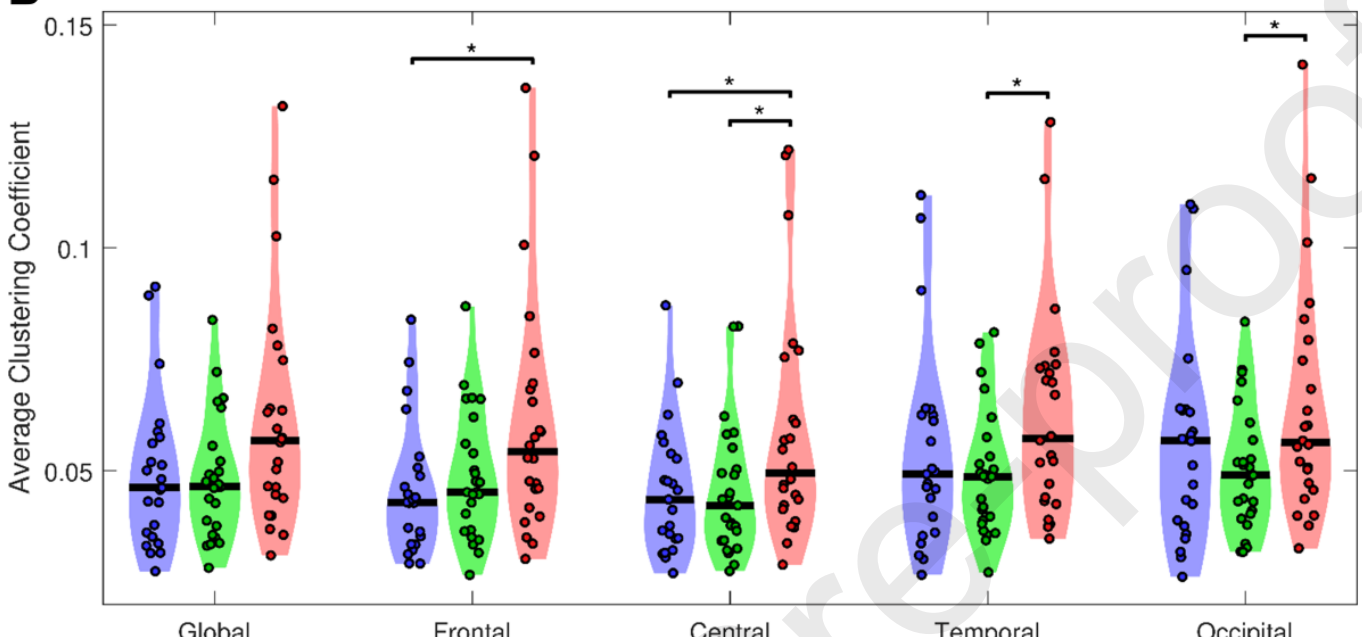

- OR

- GF

- $\mathrm{GM}$

C
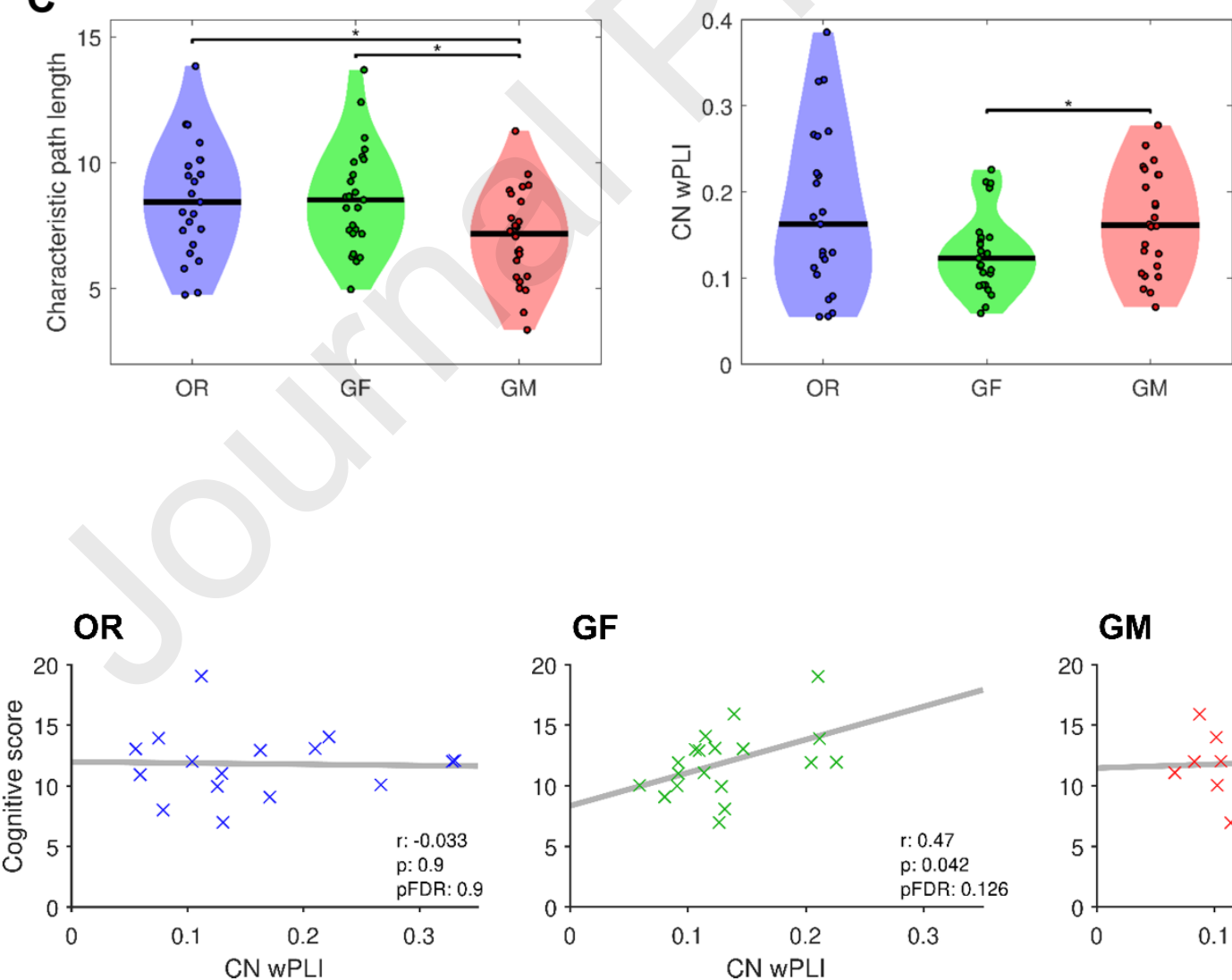

GF

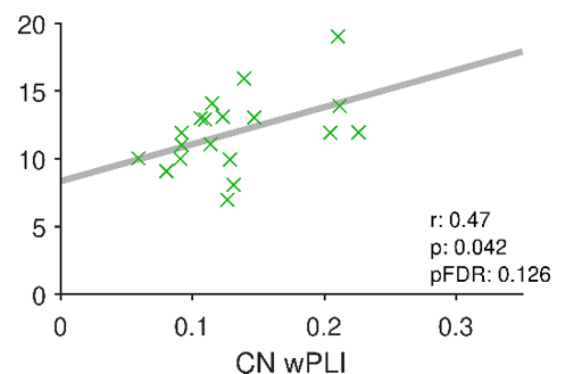

GM

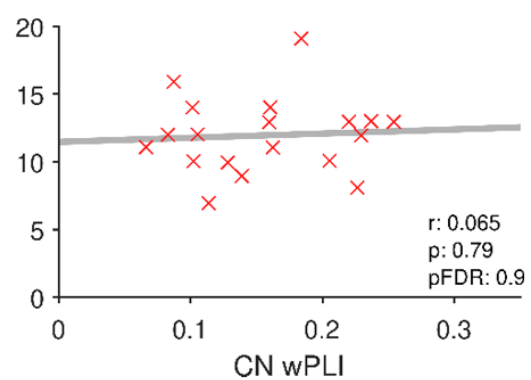

